\title{
Grain Growth in Large-Scale Molecular Dynamics Simulation: Linkage between Atomic Configuration and von Neumann-Mullins Relation
}

\author{
Shin OKITA and Yasushi SHIBUTA* \\ Department of Materials Engineering, The University of Tokyo, 7-3-1 Hongo, Bunkyo-ku, Tokyo, 113-8656 Japan. \\ (Received on July 5, 2016; accepted on August 9, 2016; J-STAGE Advance published date: October 20, \\ 2016)
}

\begin{abstract}
Grain growth in nanometer scale is closely investigated with a combination of a large-scale molecular dynamics (MD) simulation and a comprehensive post-analysis technique. The volume change of grains is directly estimated for all grains in two-dimensional and three dimensional grain growths. For the twodimensional grain growth, grains with seven and more neighboring grains generally grow larger, whereas those with five and less neighboring grains shrink and some of them disappear within the timescale of the simulation. The result agrees with the von Neumann-Mullins relation. For the three-dimensional grain growth, threshold number of neighboring grains is estimated to be approximately 14, which is close to many of reported values from previous experiments and simulations. An extended model of the vonNeumann-Mullins relation for the three-dimensional grain growth is derived based on the MacPhersonSrolovitz model, from which the threshold number of neighboring grains is estimated to be 14.7. Using the von Neumann-Mullins relation, grain boundary mobility is estimated to be in the order of $10 \times 10^{-9}$ $\mathrm{m}^{4} \mathrm{~J}^{-1} \mathrm{~s}^{-1}$, which is within the range of reported values. Results and discussion derived from the largescale MD simulation basically agree with the classical theory, which proofs the validity of simulation results from the statistical point of view, whereas most of present MD studies still limits the discussion to the local structure around of particular grain boundaries due to the size limitation. The quantitative discussion based on the large-scale MD simulation is largely attributable to the rapid progress in highperformance computational environments.
\end{abstract}

KEY WORDS: molecular dynamics simulation; grain growth; von Neumann-Mullins relation; microstructure; solidification.

\section{Introduction}

It is essential to control the microstructure of metals and alloys precisely during the production process since it affects physical and mechanical properties. Many practical metals and alloys undergo solidification and subsequent grain growth during their production. ${ }^{1)}$ Therefore, it is important to understand a series of fundamental physics ranging from nucleation and solidification to grain growth comprehensively. Although a lot of efforts have been made over years to understand fundamental aspects of microstructure evolution, ${ }^{2)}$ however, it is still challenging to control microstructures with a high degree of accuracy. One of the difficulties is to observe the evolution of threedimensional microstructure as it is during the production process. Although recent new methodologies such as synchrotron X-ray microtomography ${ }^{3)}$ and serial sectioning technique ${ }^{4)}$ achieve three-dimensional morphology of microstructure experimentally, most of observation and

* Corresponding author: E-mail: shibuta@material.t.u-tokyo.ac.jp DOI: http://dx.doi.org/10.2355/isijinternational.ISIJINT-2016-408 discussion are based on the two-dimensional cross section of the three-dimensional microstructure. Therefore, computational studies have contributed to understanding the nature of the microstructure evolution. For example, Monte Carlo (MD) simulations based on the Potts model ${ }^{5)}$ are often used to study the grain growth kinetics. ${ }^{6-10)}$ Moreover, cellular automata (CA) ${ }^{11-14)}$ front tracking method, ${ }^{15-17)}$ level-set model, ${ }^{18)}$ vertex model, ${ }^{19}$ surface-evolver model, ${ }^{20)}$ phasefield model ${ }^{21-27)}$ have been widely employed to discuss the grain growth kinetics from the mesoscale point of view. Especially, the multi-phase-field simulation ${ }^{28,29)}$ is a powerful tool to investigate microstructure evolution since it is not necessary to explicitly track the position of grain boundaries in the polycrystalline microstructure.

Although these mesoscale simulations have successfully shed light on the nature of the grain growth kinetics, there also exists a limitation in the most of mesoscale simulations. That is, there is no explicit way to treat a nucleation event. Therefore, nuclei in the melt are specified in advance as having a random (or a particular) distribution or are forcibly formed in line with an assumption in most previous phasefield and MC simulations. However, it is obvious that the 
nucleation strongly affects distributions of grain size and orientation. Therefore, it is desirable that the nucleation event naturally happens as a result of the simulation without any phenomenological parameters. Molecular dynamics (MD) simulations satisfy this request since only the interaction between neighbor atoms are given. Therefore, there are many MD studies on the nucleation over many years. In the early stage, MD studies mainly focused on the kinetic detail of nucleation via embryos formation and their aggregation in relatively small systems. ${ }^{30-35)}$ Although these studies have shed the light on a local structure of the nucleation from the atomistic point of view, it is not straightforward to discuss this matter from the statistical point of view due to the size limitation. Recent rapid progress in high-performance computational environments brings us a breakthrough. ${ }^{36}$ That is, large-scale MD simulations now cover not only the multiple nucleation in large systems ${ }^{37,38)}$ but also subsequent solidification and grain growth in a consecutive simulation, ${ }^{39-41)}$ which enables us the quantitative discussion of nucleation, solidification and grain growth from the statistical point of view. For example, the nucleation rate as a function of temperature has a characteristic shape with the nose at the critical temperature. ${ }^{39)}$ It validates that the thermallyactivated homogeneous nucleation happens spontaneously in the large-scale MD simulation without any inducing factor. Moreover, the Avrami exponents during nucleation and subsequent solidification from undercooled melt iron are approximately estimated to be close to 3 and 4 in two- and three-dimensional grain growths, respectively, which also agrees with empirical interpretation. ${ }^{40)}$

On the other hand, as the number of atoms employed in the MD simulation drastically increases, there comes another problem, that is, a heavy burden in the post-analysis. The primary information obtained from the MD simulation is only the trajectory of all atoms. In the case of the MD simulation of grain growth, there is no established way to obtain the morphology of grains directly from the trajectory of all atoms. That is, it is not obvious which atoms belong to a particular grain. Therefore, such the geometric information must be extracted manually by a combination of typical post-analysis techniques such Volonoi analysis, common-neighbor analysis, cluster analysis and so on, which is a tough work especially for the case of the large system. To overcome this problem, we have developed a comprehensive post-analysis technique ${ }^{42)}$ to pick up the geometric information of grains from the trajectory of all atoms in the MD simulation using a visualization tool, OVITO (Open visualization tool). ${ }^{43)}$ In this study, two-dimensional and three-dimensional grain growths in nanometer scale are investigated by a combination of a large-scale MD simulation and a comprehensive post-analysis technique of atomic configuration. In particular, the volume change of each grains during the grain growth is closely investigated and compared with classical models. Moreover, an extended model for the three-dimensional grain growth in nanometer scale is derived based on the MacPherson-Srolovitz model.

\section{Simulation Methodology}

\subsection{Molecular Dynamics Simulation}

The simulation methodology basically follows previ- ous studies. ${ }^{39,40)}$ The Finnis-Sinclair (FS) potential ${ }^{44)}$ is employed for the interatomic interaction between iron atoms, which is one of the most established potentials for body-centered-cubic (bcc) metals. It has been confirmed that the FS potential can reproduce concurrent nucleation and solidification from the undercooled iron melt and subsequent grain growth properly. ${ }^{39,40,45,46)}$ The total energy of the FS potential, $E$, is expressed as follows:

$$
\begin{aligned}
& E=\frac{1}{2} \sum_{i} \sum_{j} V_{i j}\left(r_{i j}\right)-A \sum_{i} \sqrt{\rho_{i}} \\
& V_{i j}\left(r_{i j}\right)=\left\{\begin{array}{ll}
\left(r_{i j}-c\right)^{2}\left(c_{0}+c_{1} r_{i j}+c_{2} r_{i j}^{2}\right) & \left(r_{i j} \leq c\right) \\
0 & \left(r_{i j}>c\right)
\end{array} \ldots \ldots . .\right. \\
& \rho_{i}=\sum_{j \neq i} \phi\left(r_{i j}\right) \\
& \phi\left(r_{i j}\right)=\left\{\begin{array}{ll}
\left(r_{i j}-d\right)^{2}+\beta \frac{\left(r_{i j}-d\right)^{3}}{d} & \left(r_{i j} \leq d\right) \\
0 & \left(r_{i j}>d\right)
\end{array},\right.
\end{aligned}
$$

where $V$ is the repulsive term, $r_{i j}$ is the bond length between atoms $i$ and $j, \rho$ is the total electronic charge density at the site of atom $i$, which is constructed by the rigid superposition of atomic charge densities $\phi, A$ is the binding energy, $c_{0}, c_{1}$, and $c_{2}$ are the free parameters used for fitting experimental data, $c$ and $d$ are cutoff parameters assumed to lie between the second-nearest- and third-nearest-neighbor atoms, $\beta$ is a parameter used to introduce a maximum value of $\phi$ within the first-nearest-neighbor distance. The parameters for bcc iron from the original FS paper are employed, ${ }^{44)}$ which are listed in Table 1. A leapfrog method is used to integrate the classical equation of motion with a time step of $5.0 \mathrm{fs}$ (energy conservancy has been proven in previous NVE constant simulations). A Berendsen thermostat ${ }^{47)}$ is applied to control the temperature in each step, and the Andersen method ${ }^{48)}$ is applied to independently control the pressure in each direction. The main calculations are carried out on the GPU (Graphics Processing Unit) supercomputer, TSUBAME2.5 at Tokyo Institute of Technology with the original code developed in CUDA (Compute Unified Device Architecture). ${ }^{49)}$

In this study, the grain growth in the quasi-twodimensional and three-dimensional systems are examined. For the quasi-two-dimensional system, simulation results from our previous study ${ }^{39)}$ on the homogeneous nucleation and solidification in the quasi-two-dimensional system (1 037880 atoms in the cell of $53.4 \times 53.4 \times 4.3 \mathrm{~nm}$ ) are employed and analyzed by a comprehensive post-analysis technique explained below. For the three-dimensional grain growth, approximately cubic system (12 454560 atoms

Table 1. Potential parameters of iron for the Finnis-Sinclair potential. $^{44)}$

\begin{tabular}{ccccccc}
\hline$d[\AA]$ & $A[\mathrm{eV}]$ & $\beta$ & $c[\AA]$ & $c_{0}$ & $c_{1}$ & $c_{2}$ \\
\hline 3.569745 & 1.828905 & 1.8 & 3.40 & 1.2371147 & -0.3592185 & -0.0385607 \\
\hline
\end{tabular}


in the cell of $53.4 \times 53.4 \times 51.6 \mathrm{~nm})$ is employed. As the calculation procedure, the initial configuration of the calculation system is prepared by heating a bcc crystal of iron at $3500 \mathrm{~K}$ for $10 \mathrm{ps}$ in a NVT ensemble. The prepared initial configuration is annealed isothermally in the main calculation for 4200 ps under zero pressure with the NPT constant ensemble at 1400 and $1200 \mathrm{~K}$, respectively. Since the melting point of bcc iron given by the FS potential, $T_{\mathrm{m}}=$ $2400 \mathrm{~K}^{50)}$ is higher than the experimental value of $1811 \mathrm{~K}$, temperatures normalized by $T_{\mathrm{m}}$ are employed. That is, 1400 and $1200 \mathrm{~K}$ correspond to $0.58 T_{\mathrm{m}}$ and $0.50 T_{\mathrm{m}}$, respectively. Visualization of the atomistic configuration and post-analyses are performed using OVITO (Open visualization tool). ${ }^{43)}$

\subsection{Comprehensive Post-analysis Technique to Obtain Information of Grains}

A comprehensive post-analysis is performed to extract the geometric information of grains from the trajectory of all atoms in the MD simulation, which basically follows the previous study. ${ }^{42)}$ Firstly, a Voronoi analysis of the whole system is performed to derive the atomic volumes. Then, an adaptive common neighbor analysis $(\mathrm{a}-\mathrm{CNA})^{51)}$ with variable cutoff distance is used to identify the local structural environment, which results in FCC (face centered cubic), HCP (hexagonal close-packed), BCC (body centered cubic). ICO (icosahedral) and unknown ("other") coordination structures. After removing atoms with unknown coordination structure, two consecutive coordination analyses with cutoff radius 0.54 and $0.35 \mathrm{~nm}$ are performed to define the coordination number of remaining atoms. Atoms with a coordination number smaller than 45 (for cutoff radius 0.54 $\mathrm{nm}$ ) and 5 (for cutoff radius $0.35 \mathrm{~nm}$ ) are removed in turn after each coordination analysis. Finally, a cluster analysis with a cutoff radius of $0.29 \mathrm{~nm}$ is used to determine the effective number of grains. The atomic volumes of the remaining atoms are summed up to obtain the Volume $V$ for each individual grain. Due to the removal of atoms after coordination analysis this is a conservative estimate close to the real grain volume. The estimated volume is converted into the effective radius $r$ for all grains under the assumption that the grain is spherical using the relationship $V=$ $4 \pi r^{3} / 3$. In this study, the effective radius is adopted as the grain size. Regarding the count of number of neighboring grains, all atoms in a target grain and atoms within $2 \mathrm{~nm}$ from the target grain are picked up and a cluster analysis is again used to count the neighboring grains. Due to the inher- ent error by picking up atoms within a cutoff radius, small grains consisting of less than 15 atoms are not regarded as neighboring grains. Schematic image of these processes are shown in Fig. 1.

\section{Results and Discussion}

\subsection{Von Neumann-Mullins Relation for Two-dimensional Grain Growth}

Firstly, two-dimensional grain growth is examined by analyzing the MD simulation results from our previous study on the homogeneous nucleation and solidification in the quasi-two-dimensional system. ${ }^{39)}$ Figure 2 shows snapshots of atomic configuration between 500 and 4000 ps of the calculation for the grain growth at $0.58 T_{\mathrm{m}}$ in the quasi-two-dimensional system. In the snapshots, most of atoms are identified as BCC (body centered cubic) and unknown ("other") coordination structure configurations. Here, most of atoms with the unknown coordination structure corresponds to that in the grain boundary. At 500

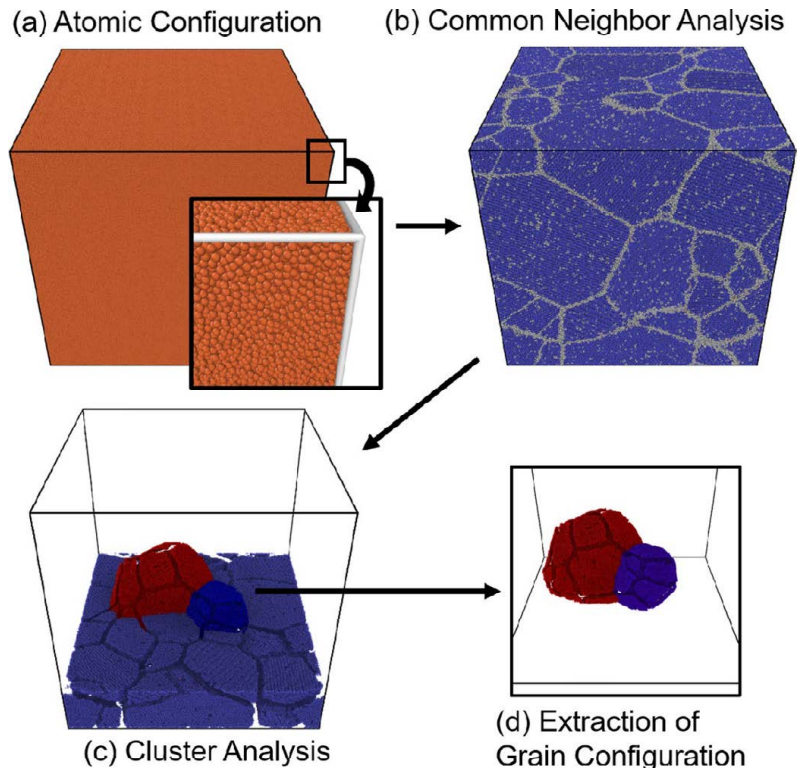

Fig. 1. Schematic image of the comprehensive post-analysis technique: (a) atomistic configuration obtained from a large-scale molecular dynamics simulation, (b) common neighbor analysis to identify the local structural environment, (c) cluster analysis to determine the grains and (d) extraction of atomistic configuration of particular grains. (Online version in color.)

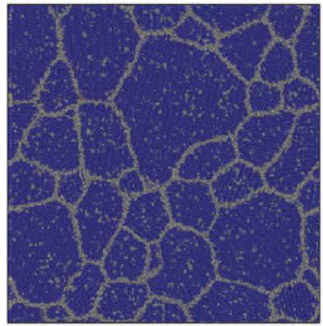

500 ps

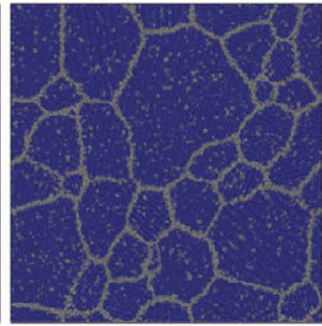

1000 ps

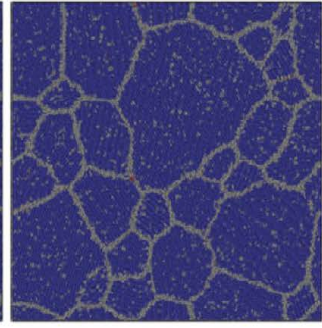

2000 ps

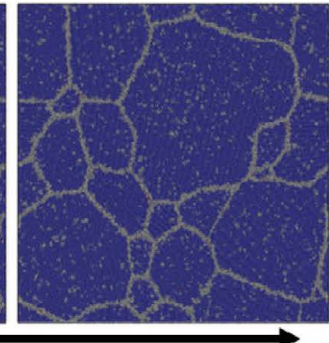

$4000 \mathrm{ps}$

Fig. 2. Snapshots of atomic configuration between 500 and $4000 \mathrm{ps}$ of the calculation for the grain growth at $0.58 T_{\mathrm{m}}$ in the quasi-two-dimensional system. ${ }^{39)}$ Blue, yellow, red, green and white atoms represent atoms with BCC, ICO, HCP, FCC and unknown coordination structure ("other") configurations, which are defined by the adaptive common neighbor analysis. (Online version in color.) 
ps, approximately 35 grains appear, which are formed by homogenous nucleation and subsequent solidification from the undercooled melt. ${ }^{39)}$ As time goes by, most of small grains shrink and some of them disappear before 4000 ps, whereas large grains become larger during the simulation. Approximately 20 grains survive at 4000 ps.

Figure 3 shows snapshots of atomic configuration between 1000 and 3500 ps of the calculation for the grain growth at $0.58 T_{\mathrm{m}}$ focusing on a particular grain (labelled 'A') contacting four grains. It is confirmed from the snapshots that Grain A shrinks as time goes by and disappears at $3500 \mathrm{ps}$. In the same manner, the volume change of all grains in the snapshot is estimated by the comprehensive post-analysis technique. Figure 4 shows the volume change of all grains during the two-dimensional grain growth as a function of the number of neighbor grains per grain $n$ for $0.58 T_{\mathrm{m}}$ and $0.50 T_{\mathrm{m}}$. The volume change during $200 \mathrm{ps}$ is measured at $1000,2000,3000$ and 4000 ps. The average volume changes for each $n$ are also plotted with error bars showing the standard deviation. It is confirmed from the graph that the volume change is proportional to the number of neighboring grains per grain and the value changes from negative to positive as the number of neighbor grains increases in both cases. In Fig. 3, lines are fitted to all plots with a least squares approximation. From the intersection of the fitted line with the line of zero volume changes, threshold numbers of neighbor grains dividing growth and shrink of grains are estimated to be 5.80 for $0.58 T_{\mathrm{m}}$ and 5.90 for $0.50 T_{\mathrm{m}}$, respectively.

It is well known that all individual grains should satisfy the von Neumann-Mullins relation ${ }^{52,53)}$ during the twodimensional ideal grain growth:

$$
\frac{d A_{n}}{d t}=\frac{\pi}{3} m \sigma(n-6),
$$

where $A_{n}$ is the area of a grain with $n$ neighbor grains, $m$ is the grain boundary mobility and $\sigma$ is the grain boundary (a) $0.58 \mathrm{~T}_{\mathrm{m}}$
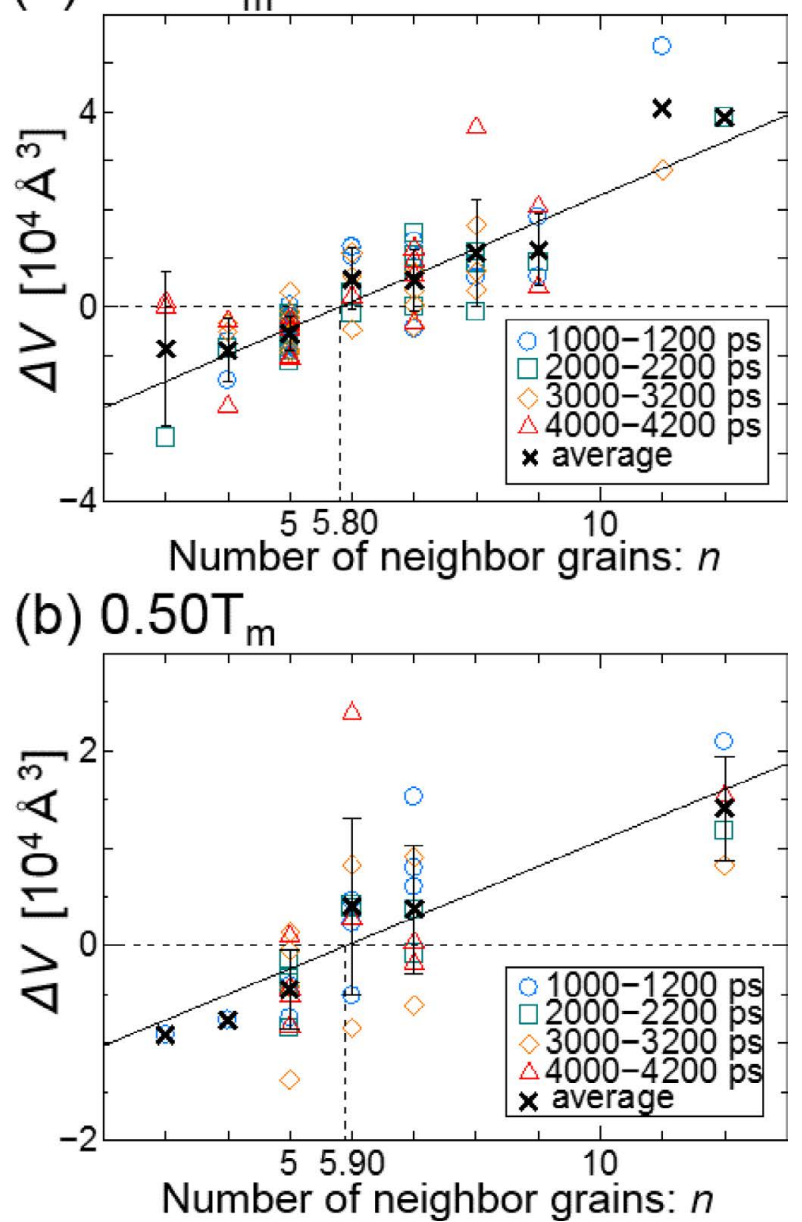

Fig. 4. Volume change of all grains during the two-dimensional grain growth for (a) $0.58 T_{\mathrm{m}}$ and (b) $0.50 T_{\mathrm{m}}$ as a function of the number of neighbor grains per grain $n$. Volume changes during 200 ps at $1000,2000,3000$ and 4000 ps of the calculations are employed. The average volume changes for each $n$ are also plotted with error bars showing the standard deviation. Lines are fitted to all plots with a least squares approximation. (Online version in color.) (a)

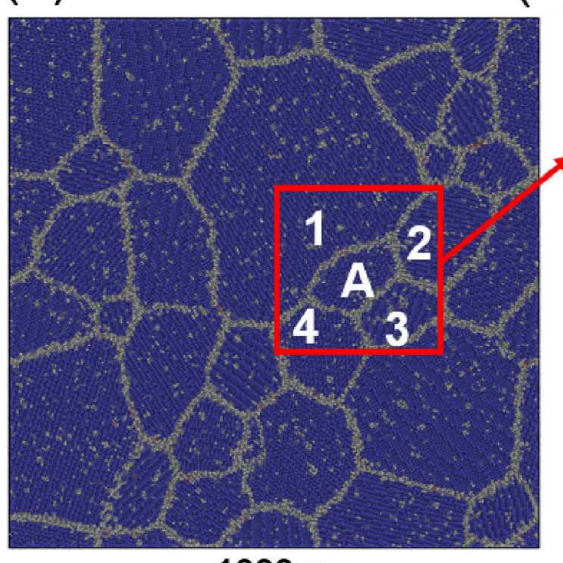

$1000 \mathrm{ps}$ (b)

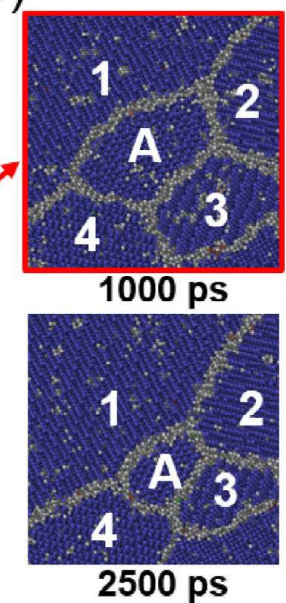

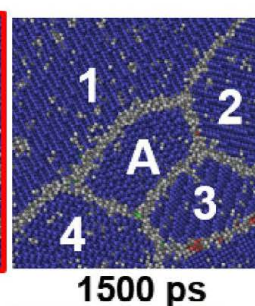
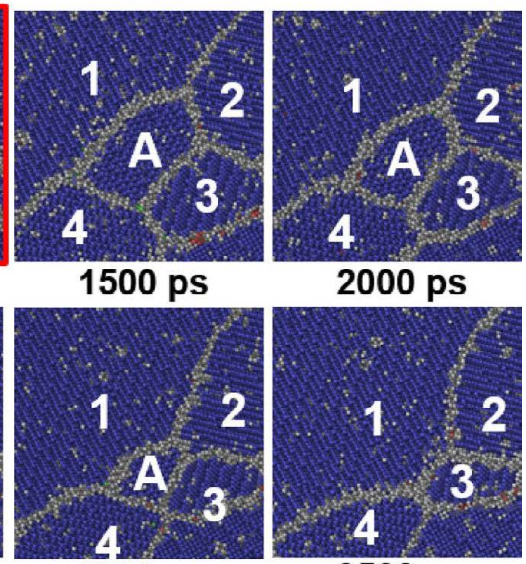

3000 ps

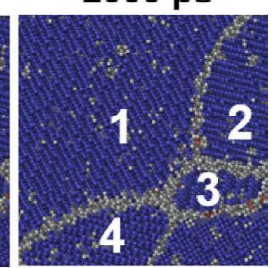

3500 ps

Fig. 3. (a) Snapshot of of atomic configuration at $1000 \mathrm{ps}$ of the calculation for the grain growth at $0.58 T_{\mathrm{m}}$ in the quasitwo-dimensional system. ${ }^{39)}$ One particular grain is labeled ' $A$ ' and four grains contacting to the Grain A are labeled '1', '2', '3' and '4'. (b) Enlarged views of snapshots focusing on Grain A between 1000 and 3500 ps of the calculation. Blue, yellow, red, green and white atoms represent atoms with BCC, ICO, HCP, FCC and unknown coordination structure ("other") configurations, which are defined by the adaptive common neighbor analysis. (Online version in color.) 
energy, respectively. That is, a grain contacting more than six grains grows larger and that contacting less than six grains shrinks. In our MD simulation with the quasi-twodimensional system, ${ }^{40)}$ only two-dimensional grain growth with the disk-like grain happens. Therefore, the volume change of grains in the quasi-two-dimensional system is equivalent to the area change. Since threshold numbers of neighboring grains estimated from the MD Simulation are close to six, it is considered that the grain growth in our MD simulation with the quasi-two-dimensional system basically satisfies the von Neumann-Mullins relation. Although the von Neumann-Mullins relation is originally derived for the ideal case without any anisotropy effect in grain boundary energy and grain boundary mobility, our result agrees well with the von Neumann-Mullins relation. It is expected that the anisotropy effect is diminished by averaging the effect from many grain boundaries since the anisotropy in the grain boundary energy of pure iron is not so large ${ }^{50)}$ except for a particular tilt grain boundary.

Moreover, it is expected from Eq. (5) that the slope of fitted lines in Fig. 3 is proportional to the product of grain boundary mobility and the grain boundary energy. The values of grain boundary mobility for $0.58 T_{\mathrm{m}}$ and $0.50 T_{\mathrm{m}}$ are roughly estimated to be $6.48 \times 10^{-9}$ and $3.12 \times 10^{-9}$ $\mathrm{m}^{4} \mathrm{~J}^{-1} \mathrm{~s}^{-1}$ assuming the grain boundary energy to be 1.0 $\mathrm{Jm}^{-2}{ }^{50)}$ It is within the range of reported values for metals and alloys $\left(10^{-8}\right.$ to $\left.10^{-10} \mathrm{~m}^{4} \mathrm{~J}^{-1} \mathrm{~s}^{-1}\right){ }^{54-58)}$ The grain bound- ary mobility for $0.58 T_{\mathrm{m}}$ is larger than that for $0.50 T_{\mathrm{m}}$, which agrees with a common sense in temperature dependence of the grain boundary mobility. ${ }^{54-58)}$ However, we note that the estimated value here includes many effects from various grain boundaries and many triple points.

\subsection{Mullins Relation for Three-dimensional Grain Growth}

Compared with the two-dimensional grain growth, it is not easy to discuss the three-dimensional grain growth. One of the difficulty is to observe the evolution of threedimensional microstructure as it is. It is not straightforward to obtain the number of neighboring grains directly during the grain growth. Therefore, most of the discussion is based on the morphology of two-dimensional cross sections of three-dimensional grains. On the other hand, we can obtain the three-dimensional microstructure directly from the MD simulation via the comprehensive post-analysis technique. Here, the number of neighboring grains during the threedimensional grain growth is directly extracted from the trajectory of atoms of the MD simulation.

Figure 5 shows snapshots of atomic configuration between 500 and 4000 ps of the calculation for the grain growth at $0.58 T_{\mathrm{m}}$ in the three-dimensional-dimensional system. At 500 ps, approximately 200 grains appear, which are formed by the homogeneous nucleation and subsequent solidification in the undercooled melt ${ }^{40,42)}$ as in the case

(a) bird's eye view
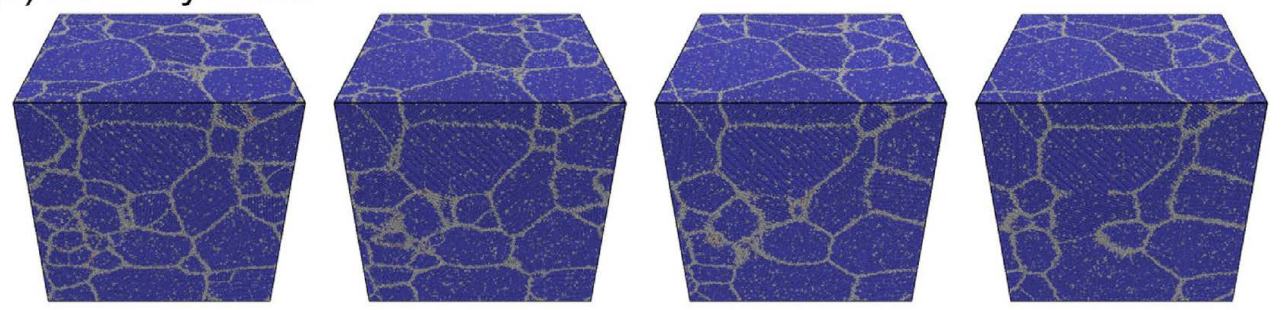

(b) cross sectional view: parallel to bottom face
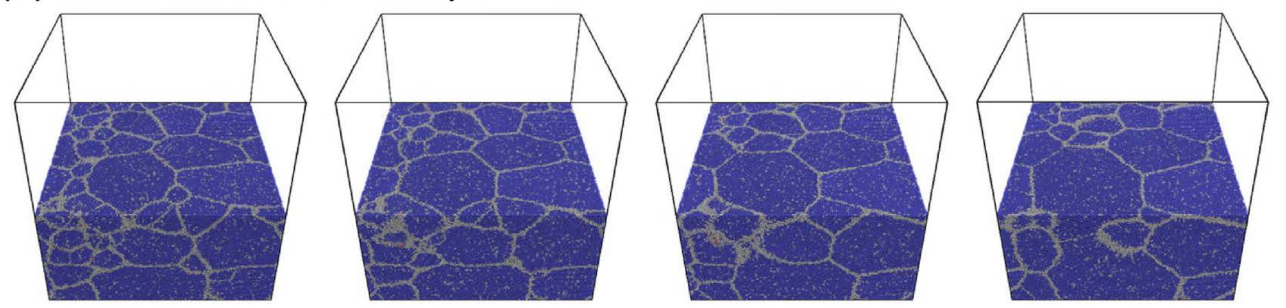

(c) cross sectional view: angled $45^{\circ}$ from bottom face

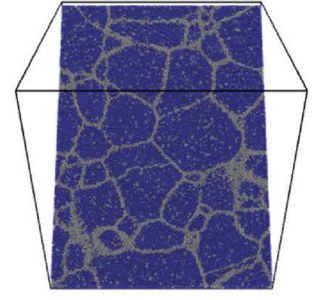

$500 \mathrm{ps}$

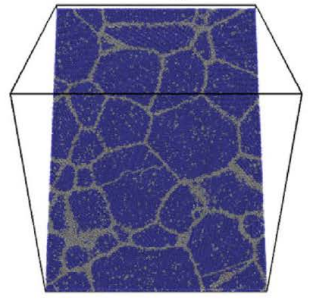

1000 ps

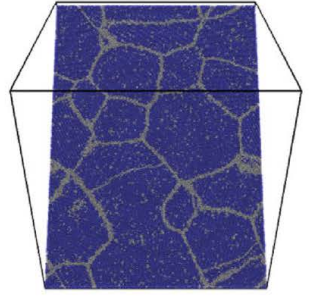

2000 ps

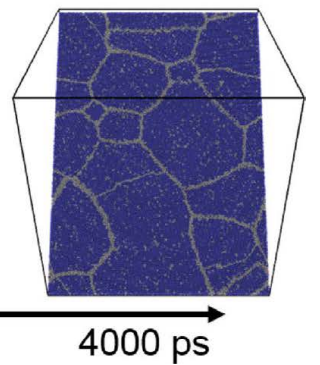

Fig. 5. Snapshots of atomic configuration between 500 and $4000 \mathrm{ps}$ of the calculation for the grain growth at $0.58 T_{\mathrm{m}}$ in the three-dimensional system. (a) Bird's eye view. (b) Cross sectional views cut by a plane parallel to the bottom face of the simulation cell. (c) Cross sectional views cut by a plane angled $45^{\circ}$ from the bottom face of the simulation cell. Blue, yellow, red, green and white atoms represent atoms with BCC, ICO, HCP, FCC and unknown coordination structure ("other") configurations, which are defined by the adaptive common neighbor analysis. (Online version in color.) 
of the quasi-two-dimensional system. As time goes by, small grains shrink and some of them disappear within the timescale of the simulation, whereas large grains become larger. From the cross-sectional views, it is confirmed that three-dimensional grain growth surely happens in the MD simulation. Figure 6 shows snapshots of atomic configuration of two contacting grains between 500 and 4000 ps of the calculation for the grain growth at $0.58 T_{\mathrm{m}}$ in the threedimensional system. Two grains are labeled 'Grain 1' and 'Grain 2' in the snapshot. The numbers of neighboring grains for Grain $1\left(n_{1}\right)$ are 25, 18, 17 and 21 at 500, 1000 , 2000 and $4000 \mathrm{ps}$, respectively, and those for Grain 2 $\left(n_{2}\right)$ are $16,14,13,12$, respectively. It is confirmed from the snapshot that Grain 1 becomes larger during the 4000 ps calculation, whereas Grain 2 becomes smaller. In the same manner, volume changes of all grains during the MD simulation in the three-dimensional cell are estimated by the comprehensive post-analysis technique. Figure 7 shows the volume change for each grain during the three-dimensional grain growth as a function of number of neighbor grains per grain $n$. Here, values of the volume change divided by cube root of the grain volume, $V^{-1 / 3} \Delta V$ are plotted in order to compare the Mullins relation for three dimensional grain growth. ${ }^{59)}$ As in the case of Fig. 3, volume changes during 200 ps at $1000,2000,3000$ and 4000 ps are plotted. The average volume changes for each $n$ are also plotted with error bars showing the standard deviation. As a whole, values of $V^{-1 / 3} \Delta V$ increase monotonically although there are variation to some extent. Therefore, values of $V^{-1 / 3} \Delta V$ change from negative to positive as the number of neighboring grains increases. The threshold number of neighboring grains dividing growth and shrink is estimated to be approximately 14 for both $0.58 T_{\mathrm{m}}$ and $0.50 T_{\mathrm{m}}$.

The general form of the three-dimensional von Neumann relation takes the following form, ${ }^{23)}$

$$
V^{-1 / 3} \frac{d V}{d t}=2 m \sigma G(n)
$$

(a) $0.58 T_{m}$

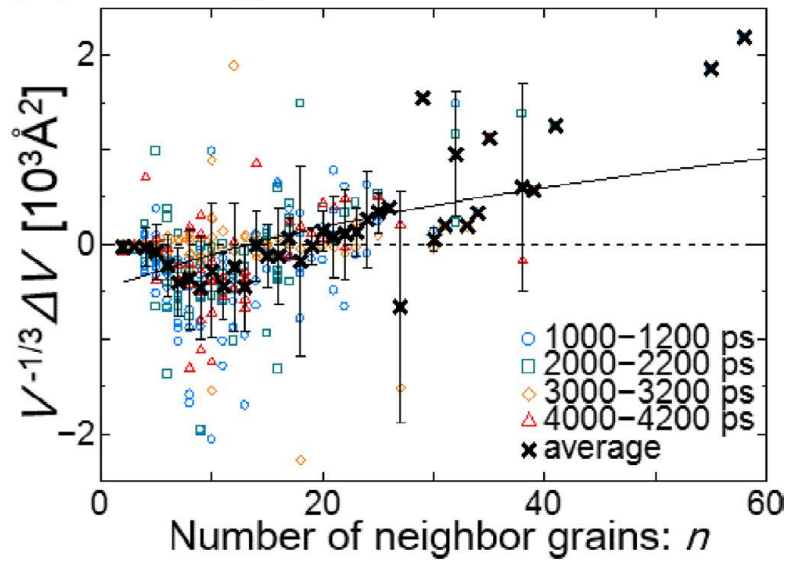

(b) $0.50 \mathrm{~T}_{\mathrm{m}}$

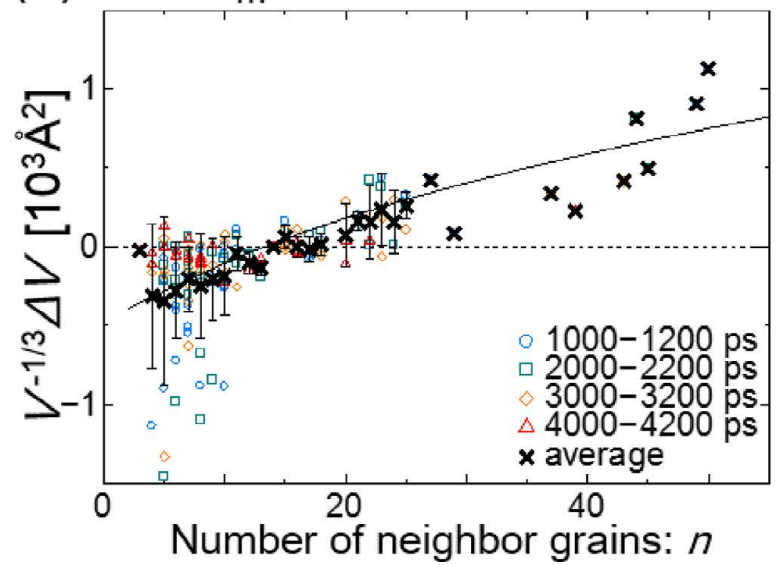

Fig. 7. Volume change for each grain during the threedimensional grain growth for (a) $0.58 T_{\mathrm{m}}$ and (b) $0.50 T_{\mathrm{m}}$ as a function of number of neighbor grains per grain $n$. Here, values of volume change divided by cube root of the grain volume, $V^{-1 / 3} \Delta V$ are plotted to compare the Mullins relation for three-dimensional grain growth. ${ }^{59)}$ The curve with the form of the Mullins relation (Eqs. (6) to (9)) are fitted to all plots with a least square approximation. (Online version in color.)

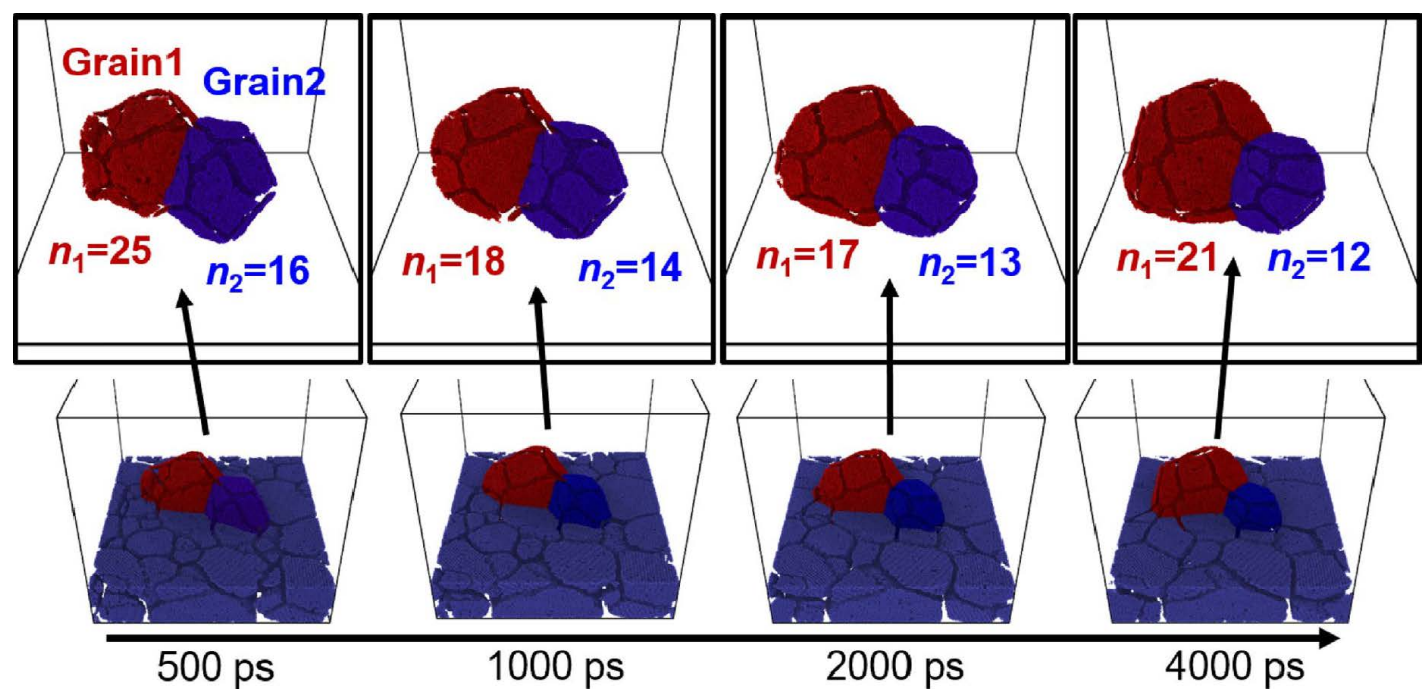

Fig. 6. Snapshots of atomic configuration of two contacting grains between 500 and 4000 ps of the calculation for the grain growth at $0.58 T_{\mathrm{m}}$ in the three-dimensional-dimensional system, which are extracted by the comprehensive post-analysis described in Section 2. Two grains are labeled 'Grain 1' and 'Grain 2' in the snapshot and $n_{1}$ and $n_{2}$ represent numbers of neighboring grains for Grain 1 and Grain 2, respectively. (Online version in color.) 
where $V$ is the grain volume. Mullins ${ }^{59)}$ proposed the function $G(n)$ as

$$
\begin{gathered}
G(n)=\frac{1}{2}\left(\frac{3}{4 \pi}\right)^{1 / 3} G_{1}(n) G_{2}(n), \ldots \ldots \ldots . \\
G_{1}(n)=\frac{\pi}{3}-2 \tan ^{-1}\left(\frac{1.86(n-1)^{1 / 2}}{n-2}\right), \ldots \\
G_{2}(n)=5.35 n^{2 / 3}\left(\frac{n-2}{2(n-2)^{\frac{1}{2}}}-\frac{3}{8} G_{1}(n)\right)^{-1 / 3}
\end{gathered}
$$

The curves are fitted to all plots in Fig. 6 using the relation of Eqs. (6) to (9) with a least square approximation. However, it is difficult to make a good fitting using this relation since the threshold number of neighboring grains is fixed to be 13.45 in the Mullins relation for three-dimensional grain growth, which is a lower than the estimated value from our simulation. Actually, many of reported values are higher than the predicted value from the Mullins relation: for example, 13.7 (Suwa et al. ${ }^{24)}$ ) and 14.7 (Kim et al. ${ }^{23)}$ ) from phase-field simulations, 15 from the surface-evolver model $^{20)}$ and 15.5 from the experimental measurement. ${ }^{60)}$ In general, it is considered that the threshold number of neighboring grains for three-dimensional grain growth is closely related to the regular packing of space filling objects with the Weaire-Phelan structure, ${ }^{61)}$ where the ideal surface-areato-volume ratio occurs for a structure consisting of one 12-sided polygon and one 14-sided polygon. However, most of reported values deviate from the ideal value, actual grains during the grain growth are not ideal polygons.

\subsection{MacPherson-Srolovitz model for Three-dimensional Grain Growth}

Recently, MacPherson and Srolovitz ${ }^{62)}$ proposed the von Neumann-Mullins relation generalized to coarsening of three-dimensional microstructure as:

$$
\frac{d V}{d t}=-2 \pi m \sigma\left(L-\frac{1}{6} \sum_{i=1}^{n} e_{i}\right)
$$

where $L$ is the mean width of the grain and $\sum_{i=1}^{n} e_{i}$ is the is the summation of the triple line lengths $e_{i}$, of the grain. Assuming the grain is spherical, the mean width is regarded as twice its diameter ${ }^{57)}$ (i.e., quadruple its radius). Therefore, Eq. (10) can be rewritten as:

$$
\frac{d V}{d t}=-2 \pi m \sigma\left(4 r-\frac{1}{6} \sum_{i=1}^{n} e_{i}\right) \equiv-2 \pi m \sigma f\left(r, e_{1}, e_{2}, \cdots, e_{n}\right),
$$

where $r$ is the radius of the grain when the grain is assumed to be spherical. Here, the factor in the parenthesis of the right side of Eq. (11) is denoted by $f$ for simplicity. Here, the radius and the summation of the triple line lengths for each grain (i.e., the factor $f$ ) are directly extracted from our MD result by the comprehensive post-analysis technique. Figure
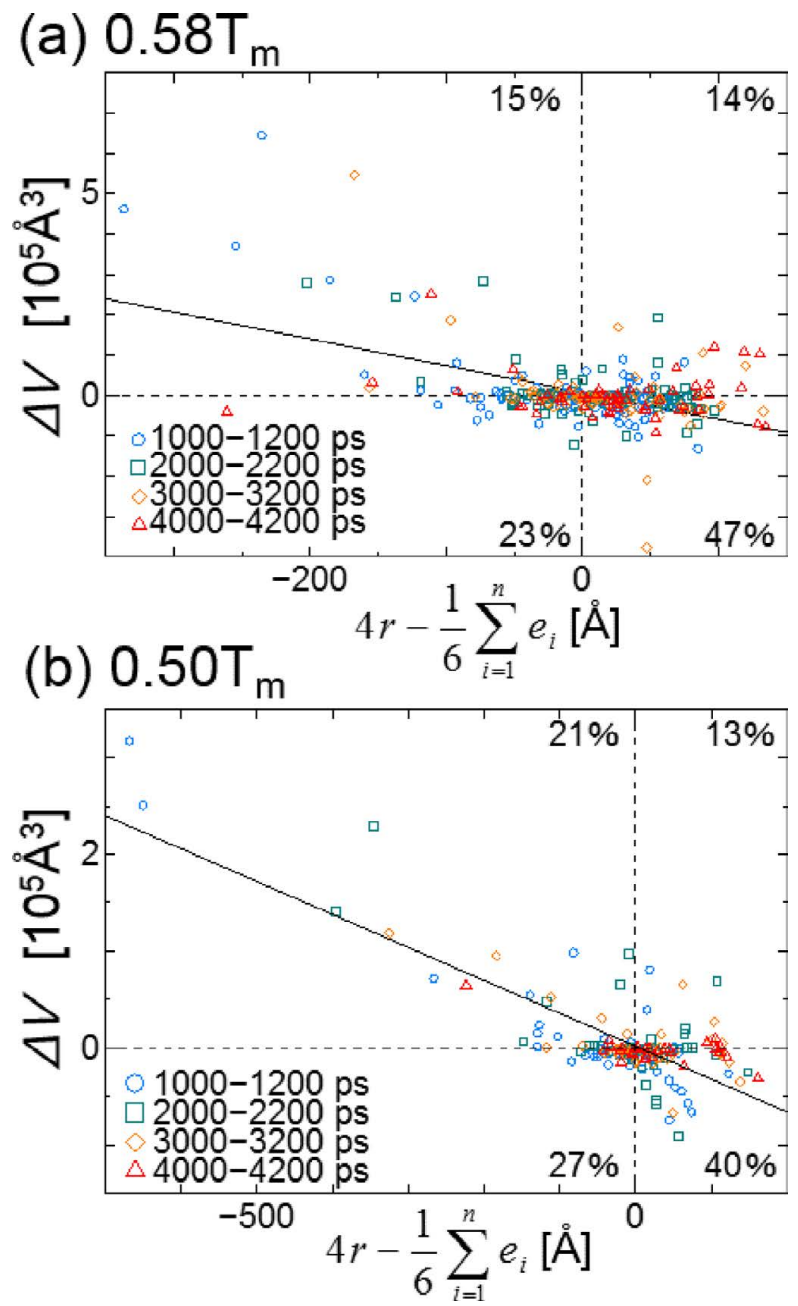

Fig. 8. Volume change for each grain during the threedimensional grain growth for (a) $0.58 T_{\mathrm{m}}$ and (b) $0.50 T_{\mathrm{m}}$ as a function of the factor, $f=4 r-\frac{1}{6} \sum_{i=1}^{n} e_{i}$, which is defined in the MacPherson-Srolovitz model ${ }^{62}$ (in Eq. (11)). Lines are fitted to all plots with a least squares approximation. (Online version in color.)

8 shows the volume change for each grain during the threedimensional grain growth as a function of the factor $f$. As a whole, the volume change is negatively correlated with the factor $f$, which is confirmed by the line fitted to all plots with a least squares approximation. The ratios of plots located in the first, the second, the third and the fourth quadrants in the graph are $14 \%, 15 \%, 23 \%$ and $47 \%$ for $0.58 T_{\mathrm{m}}$ and $13 \%$, $21 \%, 27 \%$ and $40 \%$ for $0.50 T_{\mathrm{m}}$, respectively. Since plots should exist only in the second and the fourth quadrants ideally, there are variation in data to some extent due to the inherent error with the post-analysis. Although a linearity in the volume change with respect to the factor $f$ is confirmed in Fig. 7 as expected from Eq. (11), it is not straightforward to derive the threshold number of neighboring grains during the three-dimensional grain growth directly from Eq. (11). Therefore, an extended model describing the threedimensional grain growth is derived based on the MacPherson-Srolovitz model in the following section.

\subsection{An Extended Model for Three-dimensional Grain Growth}

The factor in the parenthesis of the right side of Eq. (11) 
is rewritten using the sum of triple line length in a grain, $l$ as:

$$
4 r-\frac{1}{6} \sum_{i=1}^{n} e_{i}=4 r-\frac{l}{6}
$$

Assuming all proximal surface in a grain are circular and their areas are equal, the area of proximal surface can be defined using the radius of the proximal surface, $R$ as:

$$
\pi R^{2}=\frac{4 \pi r^{2}}{n} .
$$

Equation (13) is rewritten as,

$$
R=\sqrt{\frac{4 r^{2}}{n}}
$$

Now, $l$ can be defined as the half of the sum of circumference of all proximal surface as,

$$
l=2 \pi R \cdot n \cdot \frac{1}{2}=\pi R n
$$

By substituting Eqs. (14) and (15) into Eq. (12), following relation is obtained:

$$
4 r-\frac{l}{6}=4 r-\frac{\pi r \sqrt{n}}{3}
$$

Finally, by substituting Eq. (16) into Eq. (11), following equation is derived:

$$
\frac{d V}{d t}=-2 \pi m \sigma\left(4 r-\frac{\pi r \sqrt{n}}{3}\right) \equiv-2 \pi m \sigma g(r, n) \ldots
$$

Here, the factor in the parenthesis of the right side of Eq. (17) is denoted by $g$ for simplicity. Figure 9 shows the volume change for each grain during the three-dimensional grain growth as a function of the factor $g$. Again, the volume change is negatively correlated with the factor g, which is confirmed by the line fitted to all plots with a least squares approximation. Ratios of plots located in the first, the second, the third and the fourth quadrants in the graph are $11 \%$, $18 \%, 11 \%$ and $60 \%$ for $0.58 T_{\mathrm{m}}$ and $10 \%, 23 \%, 7 \%$ and $59 \%$ for $0.50 T_{\mathrm{m}}$, respectively. The extended model improves the classification of plots better than the MacPherson-Srolovitz model. Therefore, it is concluded that the extended model hinders the inherent error caused by the data sampling from a small system and it classifies the volume change of grains during the three-dimensional grain in nanoscale system well. The threshold number of neighboring grains is derived to be 14.6 from the extended model, which is close to many reported values as discussed in Section 3.2. Finally, it is expected from Eq. (11) that the slope of fitted lines in Fig. 8 is proportional to the product of grain boundary mobility and the grain boundary energy. The values of grain boundary mobility for $0.58 T_{\mathrm{m}}$ and $0.50 T_{\mathrm{m}}$ are roughly estimated to be $6.34 \times 10^{-9}$ and $2.69 \times 10^{-9} \mathrm{~m}^{4} \mathrm{~J}^{-1} \mathrm{~s}^{-1}$ assuming the grain boundary energy to be $1.0 \mathrm{Jm}^{-250}$ in the same matter as Section 3.2. There is no significant difference between the grain boundary mobility for two-dimensional grain growth and that for the three-dimensional growth within the spatiotemporal scale of our MD simulation. (a) $0.58 \mathrm{~T}_{\mathrm{m}}$

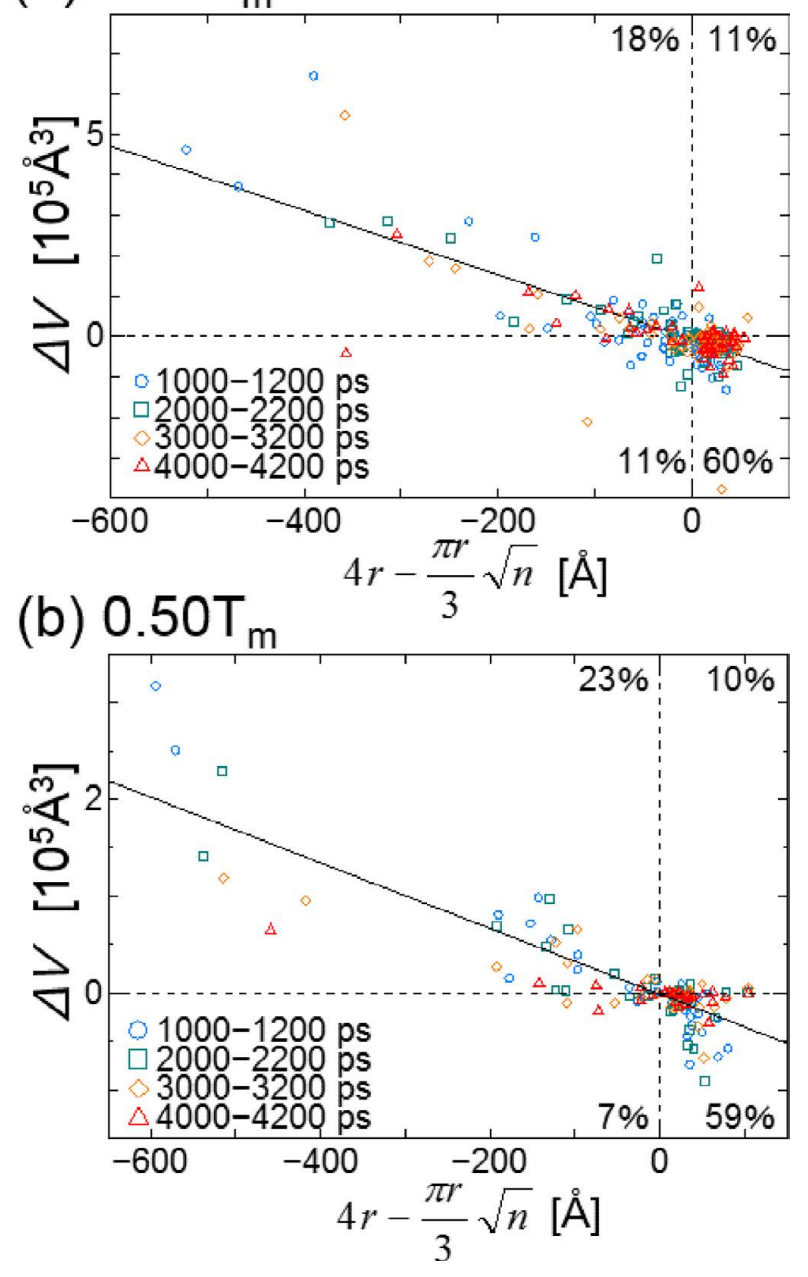

Fig. 9. Volume change for each grain during the threedimensional grain growth for (a) $0.58 T_{\mathrm{m}}$ and (b) $0.50 T_{\mathrm{m}}$ as a function of the factor, $g=4 r-\frac{\pi r \sqrt{n}}{3}$, which is defined in the extended model for three-dimensional grain growth (Eq. (17)). Lines are fitted to all plots with a least squares approximation. (Online version in color.)

\section{Conclusions}

By performing the large-scale MD simulation and the comprehensive post-analysis, two-dimensional and threedimensional grain growths in nanometer scale is closely investigated. As a whole, large grains become larger and small ones become smaller in the MD simulation over 4000 ps both in quasi-two-dimensional and three-dimensional systems. The volume change of grains during the grain growth is directly estimated by the comprehensive postanalysis. In the case of the two-dimensional grain growth, grains with seven and more neighboring grains generally grow larger, whereas those with five and less neighboring grains shrink and some of them disappear within the timescale of the simulation, which agrees with the vonNeumann-Mullins relation for the two-dimensional grain growth. In the case of the three-dimensional grain growth, the threshold number of neighboring grains dividing growth and shrink is approximately 14. Although this value is close to many of reported ones, it is a little larger than that from the Mullins relation for the three-dimensional growth. Therefore, an extended model of the von Neumann-Mullins 
relation for the three-dimensional grain growth is derived based on the MacPherson-Srolovitz model, in which the volume change is expressed as a function of grain radius and square root of the number of neighboring grains. The threshold number of neighboring grains is estimated to be 14.7 from the extended model, which is in better agreement with most of reported values than the original Mullins relation. Moreover, the grain boundary mobility is directly estimated using the von-Neumann-Mullins relation to be in the order of $10 \times 10^{-9} \mathrm{~m}^{4} \mathrm{~J}^{-1} \mathrm{~s}^{-1}$, which is within the range of many of reported values. Results and discussion derived from the large-scale MD simulation in this study basically agree with the classical theory or the common sense of a conventional material science, which proofs the validity of our results and discussion from the statistical point of view. Since most of the MD studies on the grain growth yet limits the discussion to the local structure of particular grain boundaries from the atomistic point of view due to the size limitation, it is a new insight in this study to successfully derive the relationship in the grain growth kinetics from the statistical point of view.

Finally, it is important to note that the quantitative discussion of three-dimensional grain growth in the MD simulation becomes successful for the first time owing to a combination of the large-scale MD simulation with more than ten-million atoms and the comprehensive post-analysis consisting of multiple procedures. Such the progress is largely attributable to the rapid progress in high-performance computational environments. We believe the cutting-edge approach using a GPU supercomputer is opening a new phase in computational metallurgy. Especially, the multi-GPUs parallel computation is the most promising technique, which is successful in recent large-scale simulations. ${ }^{36,63)}$ One of the remaining problems is to treat the grain growth in iron-based alloys accurately, which will be examined in the next step once the reliable interatomic potential for iron-based alloys is established.

\section{Acknowledgement}

This work was supported by Grant-in-Aid for Scientific Research (B) (No. 16H04490) from Japan Society for the Promotion of Science, and the 23rd ISIJ Research Promotion Grants from the Iron and Steel Institute of Japan. Part of this work was supported by the Joint Usage/Research Center for Interdisciplinary Large-scale Information Infrastructures (JHPCN) and the High Performance Computing Infrastructure (HPCI) in Japan for the computational environment. Authors would like to thank Prof. Munekazu Ohno, Prof. Tomohiro Takaki and Mr. Eisuke Miyoshi for fruitful discussion and Mr. Shinji Sakane for support in the CUDA coding for computing in the GPU supercomputer.

\section{REFERENCES}

1) W. Kurz and D. J. Fisher: Fundamentals of Solidification, 4th rev. ed., Trans Tech Publication, Aedermannsdorf, (1998), 13.

2) F. J. Humphreys and M. Hatherly: Recrystallization and Related Annealing Phenomena, 2nd ed., Pergamon press, Oxford, (2004), 121.

3) H. Toda, I. Sinclair, J.-Y. Buffière, E. Maire, K. H. Khor, P. Gregson and T. Kobayashi: Acta Mater., 52 (2004), 1305.

4) Y. Adachi, S. Morooka, K. Nakajima and Y. Sugimoto: Acta Mater., 56 (2008), 5995.

5) R. B. Potts: Math. Proc., 48 (1952), 106.
6) D. J. Srolovitz, M. P. Anderson, G. S. Gresta and P. S. Sahni: Scr. Metall., 17 (1983), 241.

7) M. P. Anderson, D. J. Srolovitz, G. S. Grest and P. S. Sahni: Acta Metall., 32 (1984), 783.

8) G. S. Grest, D. J. Srolovitz and M. P. Anderson: Acta Metall., 33 (1985), 509.

9) Y. Saito and M. Enomoto: ISIJ Int., 32 (1992), 267.

10) A. Ayad, F. Wagner, N. Rouag and A. D. Rollett: Comput. Mater. Sci., 68 (2013), 189.

11) H. W. Hesselbarth and I. R. Göbel: Acta Metall. Mater., 39 (1991), 2135.

12) M. Rappaz and C. A. Gandin: Acta Metall. Mater., 41 (1993), 345.

13) K. G. F. Janssens: Math. Comput. Simul., 80 (2010), 1361.

14) Y. Natsume and K. Ohsasa: ISIJ Int., 54 (2014), 415.

15) H. J. Frost, C. V. Thompson, C. L. Howe and J. Whang: Scr. Metall., 22 (1988), 65

16) A. Jacot and M. Rappaz: Acta Mater., 50 (2002), 1909.

17) E. A. Lazar, J. K. Mason, R. D. MacPherson and D. J. Srolovitz: Acta Mater., 59 (2011), 6837.

18) H. Hallberg: Model. Simul. Mater. Sci. Eng., 22 (2014), 085005.

19) A. Vondrous, M. Reichardt and B. Nestler: Model. Simul. Mater. Sci. Eng., 22 (2014), 025014.

20) F. Wakai, N. Enomoto and H. Ogawa: Acta Mater., 48 (2000), 1297.

21) L.-Q. Chen: Annu. Rev. Mater. Res., 32 (2002), 113.

22) C. E. Krill, III and L.-Q. Chen: Acta Mater., 50 (2002), 3059.

23) S. G. Kim, D. I. Kim, W. T. Kim and Y. B. Park: Phys. Rev. E, 74 (2006), 061605.

24) Y. Suwa, Y. Saito and H. Onodera: Comp. Mater. Sci., 40 (2007), 40.

25) Y. Suwa, Y. Saito and H. Onodera: Mater. Trans., 49 (2008), 704.

26) E. Miyoshi and T. Takaki: Comp. Mater. Sci., 112 (2016), 44

27) E. Miyoshi and T. Takaki: Comp. Mater. Sci., 120 (2016), 77.

28) I. Steinbach and F. Pezzolla: Physica D, 134 (1999), 385.

29) I. Steinbach, F. Pezzolla, B. Nestler, M. Seeßelberg, R. Prieler, G. J. Schmitz and J. L. L. Rezende: Physica D, 94 (1996), 135.

30) H. Pang, Z. H. Jin and K. Lu: Phys. Rev. B, 67 (2003), 094113.

31) F. J. Cherne, M. I. Baskes, R. B. Schwarz, S. G. Srinivasan and W. Klein: Model. Simul. Mater. Sci. Eng., 12 (2004), 1063.

32) J. Liu, J. Z. Zhao and Z. Q. Hu: Appl. Phys. Lett., 89 (2006), 031903.

33) Y. Shibuta and T. Suzuki: Chem. Phys. Lett., 445 (2007), 265.

34) Y. Shibuta and T. Suzuki: J. Chem. Phys., 129 (2008), 144102.

$35)$ Z. Y. Hou, L. X. Liu, R. S. Liu, Z. A. Tian and J. G. Wang: Chem. Phys. Lett., 491 (2010), 172.

36) Y. Shibuta, M. Ohno and T. Takaki: JOM, 67 (2015), 1793.

37) F. H. Streitz, J. N. Glosli and M. V. Patel: Phys. Rev. Lett., 96 (2006), 225701 .

38) R. S. Aga, J. R. Morris, J. J. Hoyt and M. Mendelev: Phys. Rev. Lett., 96 (2006), 245701

39) Y. Shibuta, K. Oguchi, T. Takaki and M. Ohno: Sci. Rep., 5 (2015), 13534.

40) Y. Shibuta, S. Sakane, T. Takaki and M. Ohno: Acta Mater., 105 (2016), 328.

41) Z. Y. Hou, K. J. Dong, Z. A. Tian, R. S. Liu, Z. Wang and J. G. Wang: Phys. Chem. Chem. Phys., 18 (2016), 17461.

42) Y. Shibuta, S. Okita, S. Sakane, T. Takaki and M. Ohno: CAMP-ISIJ, 29 (2016), 605, CD-ROM.

43) A. Stukowski: Model. Simul. Mater. Sci. Eng., 18 (2010), 015012.

44) M. W. Finnis and J. E. Sinclair: Philos. Mag. A, 50 (1984), 45

45) Y. Shibuta, K. Oguchi and T. Suzuki: ISIJ Int., 52 (2012), 2205.

46) Y. Shibuta, K. Oguchi and M. Ohno: Scr. Mater., 86 (2014), 20.

47) H. J. C. Berendsen, J. P. M. Postma, W. F. van Gunsteren, A. DiNola and J. R. Haak: J. Chem. Phys., 81 (1984), 3684.

48) H. C. Andersen: J. Chem. Phys., 72 (1980), 2384.

49) K. Oguchi, Y. Shibuta and T. Suzuki: J. Jpn. Inst. Met., 76 (2012), 462.

50) Y. Shibuta, S. Takamoto and T. Suzuki: ISIJ Int., 48 (2008), 1582.

51) A. Stukowski: Model. Simul. Mater. Sci. Eng., 20 (2012), 045021.

52) J. von Neumann: Metal Interface, ASM, Cleveland, OH, (1952), 108.

53) W. W. Mullins: J. Appl. Phys., 27 (1956), 900.

54) Y. Huang and F. J. Humphreys: Acta Mater., 47 (1999), 2259.

55) M. Upmanyu, D. J. Srolovitz, L. S. Shvindlerman and G. Gottstein: Acta Mater., 47 (1999), 3901.

56) H. Zhang, M. Upmanyu and D. J. Srolovitz: Acta Mater., 53 (2005), 79.

57) K. G. F. Janssens, D. Olmsted, E. A. Holm, S. M. Foiles, S. J. Plimpton1 and P. M. Derlet: Nat. Mater, 5 (2006), 124.

58) R. B. Godiksen, Z. T. Trautt, M. Upmanyu, J. Schiøtz, D. J. Jensen and S. Schmidt: Acta Mater., 55 (2007), 6383.

59) W. W. Mullins: Acta Metall., 37 (1989), 2979.

60) D. J. Rowenhorst, A. C. lewis and G. Spanos: Acta Mater., 58 (2010), 5511.

61) D. Weaire and R. Phelan: Philos. Mag. Lett., 69 (1994), 107

$62)$ R. D. MacPherson and D. J. Srolovitz: Nature, 446 (2007), 1053

63) T. Takaki, S. Sakane, M. Ohno, Y. Shibuta, T. Shimokawabe and T. Aoki: ISIJ Int., 56 (2016) 1427. 